\title{
Design construtal de uma cavidade aletada em forma de ' $E$ ' para refrigeração de um corpo gerador de calor
}

Constructal Design of an E-shaped cavity intruded for refrigeration of a heat generating body

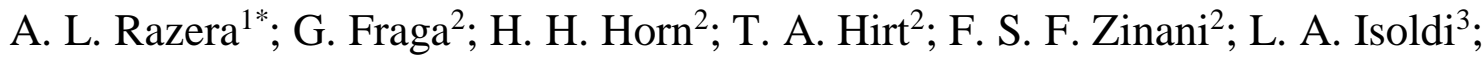 \\ E. D. dos $\operatorname{Santos}^{3} ;$ L. A. O. Rocha ${ }^{2}$ \\ ${ }^{\text {I}}$ Programa de Pós Graduação em Engenharia Mecânica, Universidade Federal do Rio Grande do Sul, 90.046-902, \\ Porto Alegre-RS, Brasil \\ ${ }^{2}$ Programa de Pós Graduação em Engenharia Mecânica, Universidade do Vale do Rio dos Sinos, 93.022-750, São \\ Leopoldo-RS, Brasil \\ ${ }^{3}$ Programa de Pós Graduação em Engenharia Oceânica, Universidade Federal do Rio Grande, 96.201-900, Rio \\ Grande-RS, Brasil
}

*razera.andre@gmail.com

\begin{abstract}
Este trabalho tem como propósito investigar através do método Design Construtal a influência geométrica de uma cavidade aletada em forma de 'E' inserida em um corpo sólido com geração interna de calor. O objetivo principal do estudo é a obtenção da geometria da cavidade que conduz a uma menor temperatura máxima no sistema. Também são obtidas configurações ótimas para diferentes frações de área entre o corpo condutor, cavidade e aleta. O sistema foi assumido como bidimensional e as condições de temperatura prescrita e convectiva foram consideradas nas paredes da cavidade e aleta. A solução numérica do problema foi obtida com a ferramenta PDETOOL, Partial Differential Equations Tool, do software MATLAB®, que resolve a equação da difusão do calor pelo método dos elementos finitos. O método Design Construtal mostrou-se adequado para a obtenção das configurações geométricas ótimas do sistema, uma vez que a temperatura máxima do corpo aquecido foi minimizada, tornando possível a obtenção de uma configuração que equilibrou a distribuição de temperaturas no sistema a favor da função objetivo, atendendo assim, aos princípios da Teoria Construtal. Além disso, constatou-se que os resultados otimizados apresentaram ganhos de performance de até $74 \%$ em relação aos casos de menor desempenho avaliados.
\end{abstract}

Palavras-chave: Cavidade aletada, Design Construtal, Resfriamento

The purpose of this work is to investigate, through the Construtal Design method, the geometric effect of an E-finned cavity inserted in a solid body with internal heat generation. The main objective of the study is to obtain cavity geometry that leads to a lower maximum temperature in the system. Optimum configurations are also obtained for different fractions of area between the conducting body, cavity and fin. The system was assumed to be two-dimensional and the prescribed and convective temperature conditions were considered in the cavity and fin walls. The numerical solution of the problem was obtained with the PDETOOL tool, Partial Differential Equations Tool, of MATLAB ${ }^{\circledR}$ software, which solves the heat diffusion equation by the finite element method. The Construtal Design method was adequate to obtain the optimal geometric configurations of the system, since the maximum temperature of the heated body was minimized, making it possible to obtain a configuration that balanced the distribution of temperatures in the system in favor of the objective function according to the principles of the Constructal Theory. In addition, it was verified that the optimized results presented performance gains of up to $74 \%$ in relation to the cases of lower performance evaluated.

Keywords: Finned cavity, Constructal Design, Cooling

\section{INTRODUÇÃO}

A transferência de calor é uma área de fundamental importância em diversos problemas de engenharia. $\mathrm{O}$ aprimoramento crescente da tecnologia fez com que o aperfeiçoamento das técnicas de transferência de calor de sistemas com geração de calor ganhasse um espaço significativo no cenário da pesquisa e desenvolvimento nas áreas da eletromecânica e eletroeletrônica.

De um modo mais específico, em equipamentos eletrônicos, a densidade de calor aumentou consideravelmente devido à minimização do volume ocupado por estes equipamentos para uma mesma capacidade de processamento e geração de energia térmica. Consequentemente, os 
desempenhos globais do sistema, bem como a segurança do mesmo, são afetados de forma significativa. Uma forma de atenuar os problemas gerados pela alta densidade de calor em equipamentos eletrônicos pode ser obtida através da inserção de cavidades e aletas nos dispositivos, aumentando a área de transferência de calor entre o equipamento aquecido e o meio em menor temperatura, produzindo assim, um efeito a favor da transferência de calor no sistema $[1,3]$.

Um modo de se obter um melhor desempenho térmico dos sistemas consiste na aplicação do método Design Construtal. O método Design Construtal é baseado na Teoria Construtal, que afirma que a geometria dos sistemas de escoamento segue um princípio físico, que é a Lei Construtal (2000) [4]. O estabelecimento da Lei Construtal (2000) [4] iniciou a partir da percepção de que o "design" dos sistemas de escoamento é um fenômeno físico universal e que pode ser entendido como uma tendência dos sistemas de escoamento em fluir através de caminhos de menor resistência. De acordo com a Lei Construtal, (2000) [4] qualquer sistema de escoamento de dimensões finitas para persistir ao longo do tempo deve evoluir sua geometria de forma a maximizar o acesso de suas correntes internas [5]. Em projetos de engenharia, os conceitos da Teoria Construtal são aplicados através do método Design Construtal, onde se definem os objetivos, restrições e graus de liberdade dos sistemas, desse modo, dando liberdade para que eles modifiquem sua geometria, visando obter a configuração geométrica que melhor distribui suas imperfeições e, a partir disso, as resistências aos fluxos presentes no sistema são minimizadas [6, 7].

Recentemente, a teoria Construtal tem sido aplicada em diversas pesquisas relacionadas ao dimensionamento de cavidades com transferência de calor, as quais apresentaram resultados satisfatórios na busca de geometrias que favoreçam uma adequada distribuição de calor em sistemas com alta densidade de calor. Biserni et al. (2004) [1] estudaram numericamente a otimização de cavidades em forma de ' $\mathrm{C}$ ' e ' $\mathrm{T}$ ' inseridas em corpos sólidos retangulares com geração de calor. $\mathrm{O}$ objetivo do trabalho foi minimizar a resistência térmica global entre o sólido e a cavidade. Do mesmo modo, Biserni et al. (2007) [2] e Lorenzini et al. (2011) [3] estudaram, respectivamente, a influência da geometria de cavidades em forma de ' $\mathrm{H}$ ' e ' $\mathrm{Y}$ ' inseridas em sólidos condutores retangulares com geração interna de calor. Ainda nesse sentido, Rocha et al. (2005) [8] avaliaram a influência da geometria de cavidades em forma de ' $C$ ', inseridas em corpos sólidos trapezoidais, enquanto Lorenzini et al. (2012) [9] exploraram a otimização de cavidades retangulares inseridas em sólidos cilíndricos com geração de calor. Além dos trabalhos citados anteriormente, pode-se destacar diversos estudos onde foram aplicados os conceitos da teoria Construtal na otimização geométrica de cavidades, aletas e caminhos de alta condutividade [10 - 16].

Deste modo, o presente trabalho busca avaliar, através do método Design Construtal associado a busca exaustiva, a geometria de cavidades aletadas em forma de ' $E$ ' inseridas em corpos sólidos condutores de formato retangular, com geração de calor uniforme. O objetivo principal do estudo é obter a geometria da cavidade que conduz a uma menor temperatura máxima no sistema. $\mathrm{O}$ sistema foi assumido como bidimensional. Nas paredes da cavidade, foram impostos dois tipos de condições de contorno: temperatura e convecção. A solução numérica foi obtida com a ferramenta PDETOOL do software MATLAB ${ }^{\circledR}$, que resolve a equação da difusão do calor pelo método dos elementos finitos.

\section{MODELAGEM MATEMÁTICA}

Neste trabalho, realizou-se um estudo numérico de uma cavidade em forma de 'E' inserida em um corpo condutor, com geração interna de calor. A Figura 1 apresenta o domínio proposto, onde é possível visualizar as características geométricas e condições de contorno do sistema. As paredes externas do sólido apresentam a condição de parede adiabática, enquanto as paredes da aleta e cavidade são estudadas através das condições de temperatura prescrita $\left(\widetilde{T}_{0}(\mathrm{Eq} .(8))=0\right)$, e convectiva $(\Lambda$ (Eq. $(13))=0,1 ; 1,0 ; 10 ; 100 ;)$. Para simplificar o estudo, a geometria foi assumida como bidimensional $(x, y)$, adotando-se a hipótese da terceira dimensão $(w)$ suficientemente longa em relação as outras dimensões do domínio $(H$ e $L)$. Além disso, o material foi considerado isotrópico, com condutividade térmica $(k)$ constante.

O método Design Construtal foi utilizado para definir as restrições, as variáveis de projeto e a função objetivo do problema. A geometria possui três restrições: a primeira é a área que inscreve o 
sólido condutor $(A)$, a segunda é a área que inscreve a cavidade dentro do sólido $\left(A_{\mathrm{c}}\right)$ e a terceira é a área ocupada pela aleta no interior da cavidade $\left(A_{\mathrm{f}}\right)$, dadas, respectivamente por:

$$
\begin{aligned}
& A=H . L \\
& A_{c}=H_{0} \cdot L_{0} \\
& A_{f}=H_{1} \cdot L_{1}
\end{aligned}
$$

Assim, a partir das Eqs. (1), (2) e (3), pode-se determinar duas relações adimensionais entre as áreas do sistema, dadas através das Eqs. (4) e (5).

$$
\begin{gathered}
\phi_{0}=\frac{A_{c}}{A} \\
\phi_{1}=\frac{A_{f}}{A_{c}}
\end{gathered}
$$

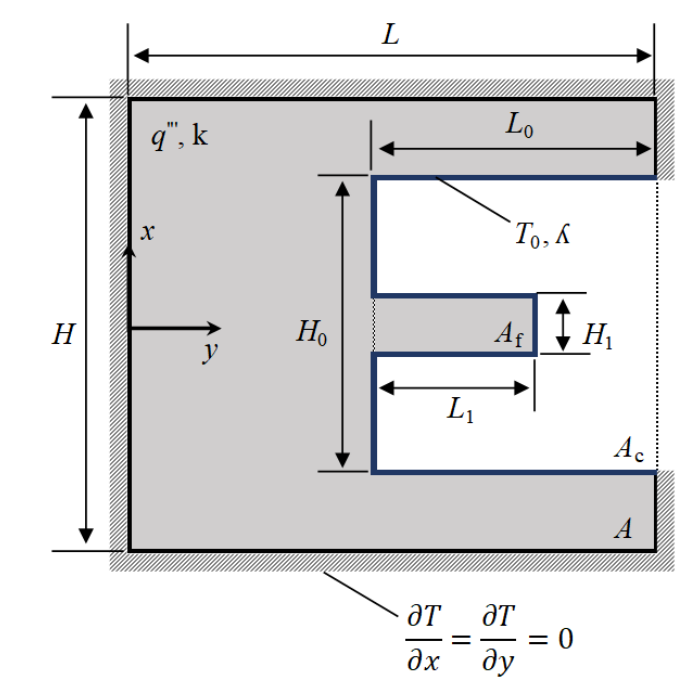

Figura 1: Domínio físico do problema e suas condições de contorno.

Desta forma, é possível definir a geometria do sistema para qualquer valor de $\phi_{0}$ e $\phi_{1}$, os quais representam respectivamente, a fração da área ocupada pela cavidade em relação a área que inscreve o sólido, e a razão entre a área ocupada pela aleta em relação a cavidade. Para este estudo, as variáveis de projeto do sistema foram definidas como: a razão entre a altura e o comprimento do corpo condutor $(H / L)$ e a razão entre a altura e o comprimento da aleta $\left(H_{1} / L_{1}\right)$, e a razão entre a altura e o comprimento da cavidade $\left(H_{0} / L_{0}\right)$. Neste trabalho, $H / L$ e $H_{1} / L_{1}$ foram admitidos como constantes, sendo seus valores igual a unidade $\left(H / L=1\right.$ e $\left.H_{1} / L_{1}=1\right)$. Além disso, a relação entre a área da aleta e a área da cavidade $\left(\phi_{1}\right)$ também teve seu valor fixado, dado por $\phi_{1}=0,1$. A relação entre a altura e o comprimento da cavidade $\left(H_{0} / L_{0}\right)$ foi a variável de projeto, e a função objetivo foi minimizar a temperatura máxima no sistema. $\mathrm{O}$ comprimento da aleta obedeceu à restrição de não se estender para além do volume da cavidade. Foram investigados diferentes valores de $\phi_{0}$.

A modelagem física do problema foi realizada através da equação do calor, a qual é tratada sobre as hipóteses de: domínio bidimensional (nas direções $x$ e $y$ ), regime permanente, geração interna de calor e propriedades termofísicas constantes, sendo definida através da Eq. (6):

$$
\frac{\partial^{2} T}{\partial x^{2}}+\frac{\partial^{2} T}{\partial y^{2}}+q^{\prime \prime \prime}=0
$$


Com o intuito de generalizar os resultados, os parâmetros do problema foram adimensionalizados. Desse modo, definiu-se os adimensionais de coordenadas e temperatura respectivamente pelas Eqs. (7) e (8):

$$
\begin{aligned}
& \widetilde{H}, \widetilde{L}, \widetilde{H_{0}}, \widetilde{L_{0}}, \widetilde{H_{1}}, \widetilde{L_{1}}=\frac{H, L, H_{0}, L_{0}, H_{1}, L_{1}}{A^{1 / 2}} \\
& \widetilde{T}=\frac{T-T_{0}}{q^{\prime \prime \prime} A / k}
\end{aligned}
$$

Dessa maneira, substituindo as variáveis adimensionais na equação do calor (Eq. (6)), obtém-se a equação adimensional do calor:

$$
\frac{\partial^{2} \tilde{T}}{\partial \tilde{x}^{2}}+\frac{\partial^{2} \tilde{T}}{\partial \tilde{y}^{2}}+1=0
$$

Do mesmo modo, as condições de contorno adimensionais aplicadas ao corpo gerador de calor são dadas respectivamente pelas Eqs. (10) (paredes externas do sólido), (11) e (12) (paredes da cavidade e aleta), para as condições de parede adiabática, temperatura prescrita e parede convectiva.

$$
\begin{aligned}
& \frac{\partial \tilde{T}}{\partial \tilde{x}}=\frac{\partial \tilde{T}}{\partial \tilde{y}}=0 \\
& \tilde{T}=\widetilde{T}_{0}=0 \\
& \frac{\partial \tilde{T}}{\partial \tilde{x}}=\frac{\partial \tilde{T}}{\partial \tilde{y}}=-\Lambda \tilde{T}
\end{aligned}
$$

onde o parâmetro $K$ é uma constante definida pela Eq. (13), e é estudado para os valores de: $\Lambda=$ 0,$1 ; 1,0 ; 10 ; 100$.

$$
\Lambda=\frac{h \cdot A^{1 / 2}}{k}
$$

\subsection{MODELAGEM NUMÉRICA}

A solução numérica da Eq. (9), sujeita às condições de contorno dadas pelas Eqs. (11), (12) e (13), foram obtidas através da ferramenta PDETOOL, do software MATLAB ${ }^{\circledR}$, a qual é fundamentada no método dos elementos finitos. Para a análise numérica, adotou-se uma malha bidimensional não-uniforme (nas direções $x$ e $y$ ) com base em elementos triangulares.

A malha adotada foi determinada por meio de refinamentos sucessivos, onde o número de elementos foi aumentado em quatro vezes para cada avaliação. Assim, pode-se estudar a qualidade da malha com o objetivo de determinar o número de elementos finitos necessários para que a malha não gere influência sobre os resultados do trabalho. A malha é considerada adequada quando o desvio relativo da temperatura adimensional máxima $\left(\tilde{T}_{\max }\right)$ entre malhas de diferentes números de elementos for menor que $0,1 \%$. A Tabela 1 mostra os resultados da análise para o sistema dado por $\phi_{0}=0,05 ; \phi_{1}=0,1 ; H / L=1 ; H_{0} / L_{0}=1 ; H_{1} / L_{1}=2$, em que os indicies $(j)$ e $(j+1)$ representam respectivamente os resultados de $\left(\tilde{T}_{\max }\right)$ para a malha avaliada e a malha de maior número de elementos.

Tabela 1: Análise de qualidade de malha para $\phi_{0}=0,05 ; \phi_{1}=0,1 ; H / L=1 ; H_{0} / L_{0}=1 ; H_{1} / L_{l}=2$.

\begin{tabular}{ccc}
\hline \hline $\begin{array}{c}\text { Número de } \\
\text { Elementos }\end{array}$ & $\widetilde{T}_{\text {max }}^{j}$ & |100. $\left(\widetilde{T}_{\text {max }}^{j}-\widetilde{T}_{\text {max }}^{j+1}\right) / \widetilde{T}_{\text {max }}^{j} \mid$ \\
\hline \hline
\end{tabular}




\begin{tabular}{ccc}
317 & 0,44889 & 1,4524 \\
1268 & 0,45541 & 0,5972 \\
5072 & 0,45813 & 0,2422 \\
$\mathbf{2 0 2 8 8}$ & $\mathbf{0 , 4 5 9 2 4}$ & $\mathbf{0 , 0 9 7 9}$ \\
81152 & 0,45969 & ----- \\
\hline
\end{tabular}

Após a realização dos testes, verificou-se que a malha de aproximadamente 20000 elementos pode ser considerada apropriada para o estudo proposto, apresentando um desvio relativo menor que $0,1 \%$ em relação a malha de maior número de elementos. Além disso, o modelo numérico adotado já foi utilizado e verificado na literatura por Biserni et al. (2004) [1].

\section{RESULTADOS E DISCUSSÃO}

Os campos de temperaturas foram obtidos com o objetivo de determinar a razão de aspecto entre a altura e a largura da cavidade $\left(H_{0} / L_{0}\right)$ que conduzem a uma menor temperatura máxima $\left(\widetilde{T}_{\text {max }}\right)$ no corpo aquecido. A razão entre a área que inscreve o corpo aquecido e a área da cavidade, $\phi_{0}$, foi estudada de acordo com: $\phi_{0}=0,05 ; 0,10 ; 0,20 ; 0,30$. Os parâmetros $\phi_{1}=0,1 ; H / L=1 ; H_{1} / L_{1}=1$ foram mantidos fixos para o conjunto de simulações realizadas. Além disso, a geometria do sistema foi avaliada para diferentes condições de contorno nas paredes da aleta e cavidade, através das condições de temperatura prescrita $\left(\widetilde{T}_{0}=0\right)$ e superfície convectiva $(\Lambda=0,1 ; 1,0 ; 10 ; 100)$. Vale destacar que a resolução utilizada para a variação do grau de liberdade $H_{0} / L_{0}$ foi de 0,1 .

Através da Figura 2, pode-se visualizar como a temperatura máxima no corpo aquecido $\left(\widetilde{T}_{\text {max }}\right)$ se comporta em função da razão de aspecto $H_{0} / L_{0}$ para diferentes valores de $\phi_{0}$. É possível observar que as curvas demonstram uma tendência de comportamento semelhante, de modo que, através da diminuição da razão de aspecto $H_{0} / L_{0}$, tem-se um decréscimo dos valores de $\tilde{T}_{\max }$ até um ponto de mínimo. Ainda, os menores valores de $\widetilde{T}_{\text {max }}$ foram obtidos para os maiores valores de $\phi_{0}$, dado por $\phi_{0}=0,3$. Observou-se também, que com o aumento de $\Lambda$, a transferência de calor no sistema foi favorecida, atingindo para a condição de $K=100$ um desempenho na transferência de calor similar a condição obtida pelo contorno de temperatura prescrita $\left(\widetilde{T}_{0}=0\right)$.

Assim, pode-se afirmar que as condições que favoreceram uma adequada distribuição de temperatura no sistema foram obtidas para os mínimos valores de $H_{0} / L_{0}$ estudados. Este comportamento pode ser explicado através da Figura 3, onde são apresentados os campos de temperaturas para os valores extremos de $H_{0} / L_{0}$ estudados $\left(H_{0} / L_{0}=0,4\right.$ e $\left.H_{0} / L_{0}=3,0\right)$ para $\phi_{0}=0,3$ e $\tilde{T}_{0}=0$. Avaliando a Figura $3 \mathrm{a}\left(H_{0} / L_{0}=0,4\right)$, nota-se que o calor se distribui de forma mais homogênea pelo sistema, evitando a ocorrência de zonas de alta temperatura no corpo aquecido. Em contrapartida, para a configuração de $H_{0} / L_{0}=3,0$, Figura $3 \mathrm{~b}$, a cavidade acaba ficando distante das extremidades do sistema, dificultando a remoção de calor em algumas regiões do corpo aquecido, e assim, ocasionando em zonas de maior temperatura máxima $\left(\tilde{T}_{\text {max }}\right)$. Deste modo, a razão de aspecto ótima entre a altura e a largura da cavidade, $\left(H_{0} / L_{0}\right)_{0}=0,4$ (para $\phi_{0}=0,3 ; \phi_{1}=0,1 ; \widetilde{T}_{0}=0$; $\left.H / L=1 ; H_{1} / L_{1}=1\right)$, conduz o sistema a ter uma redução em sua temperatura máxima de até $74 \%$ quando comparado a configuração geométrica de menor desempenho $\left(H_{0} / L_{0}=3,0\right)$.

A Figura 4a mostra o comportamento das geometrias ótimas $\left(\left(H_{0} / L_{0}\right)_{0}\right)$ obtidas para o sistema em função de diferentes valores de $\phi_{0}$. Dentre os casos avaliados, as geometrias ótimas coincidiram para os menores valores de $H_{0} / L_{0}$ estudados, para todas as condições de contorno consideradas. Além disso, pode-se notar que a curva apresenta um ligeiro crescimento na medida em que $\phi_{0}$ aumenta. Este fato ocorre pois, com o acréscimo de $\phi_{0}$ tem-se um intervalo menor de liberdade para $H_{0} / L_{0}$, assim, ocasionando em maiores valores de $\left(H_{0} / L_{0}\right)_{\mathrm{o}}$ quando maiores valores de $\phi_{0}$ forem avaliados.

Na Figura 4b, é apresentado o comportamento da temperatura máxima para os casos otimizados, $\left(\tilde{T}_{\text {max }}\right)_{\min }$, em função de $\phi_{0}$. Conforme esperado, com o acréscimo de $\phi_{0}$, foram obtidas as menores temperaturas no sistema, uma vez que, com o aumento de $\phi_{0}$, a cavidade ocupa uma maior proporção de área no corpo aquecido, e assim, ocasionando em uma maior remoção de calor. Esta característica pode ser visualizada através da Figura 5, onde são mostrados os campos de 
temperatura obtidos para diferentes valores de $\phi_{0}$, considerando-se: $\widetilde{T}_{0}=0 ; \phi_{1}=0,10 ; H / L=1$; $H_{1} / L_{1}=1 ; H_{0} / L_{0}=0,40$. É também importante notar na Figura 5 que a temperatura máxima se distribui otimamente a medida que o valor $\phi_{0}$ aumenta estando de acordo com o princípio da ótima distribuição das imperfeições conforme Bejan (2000) [4].
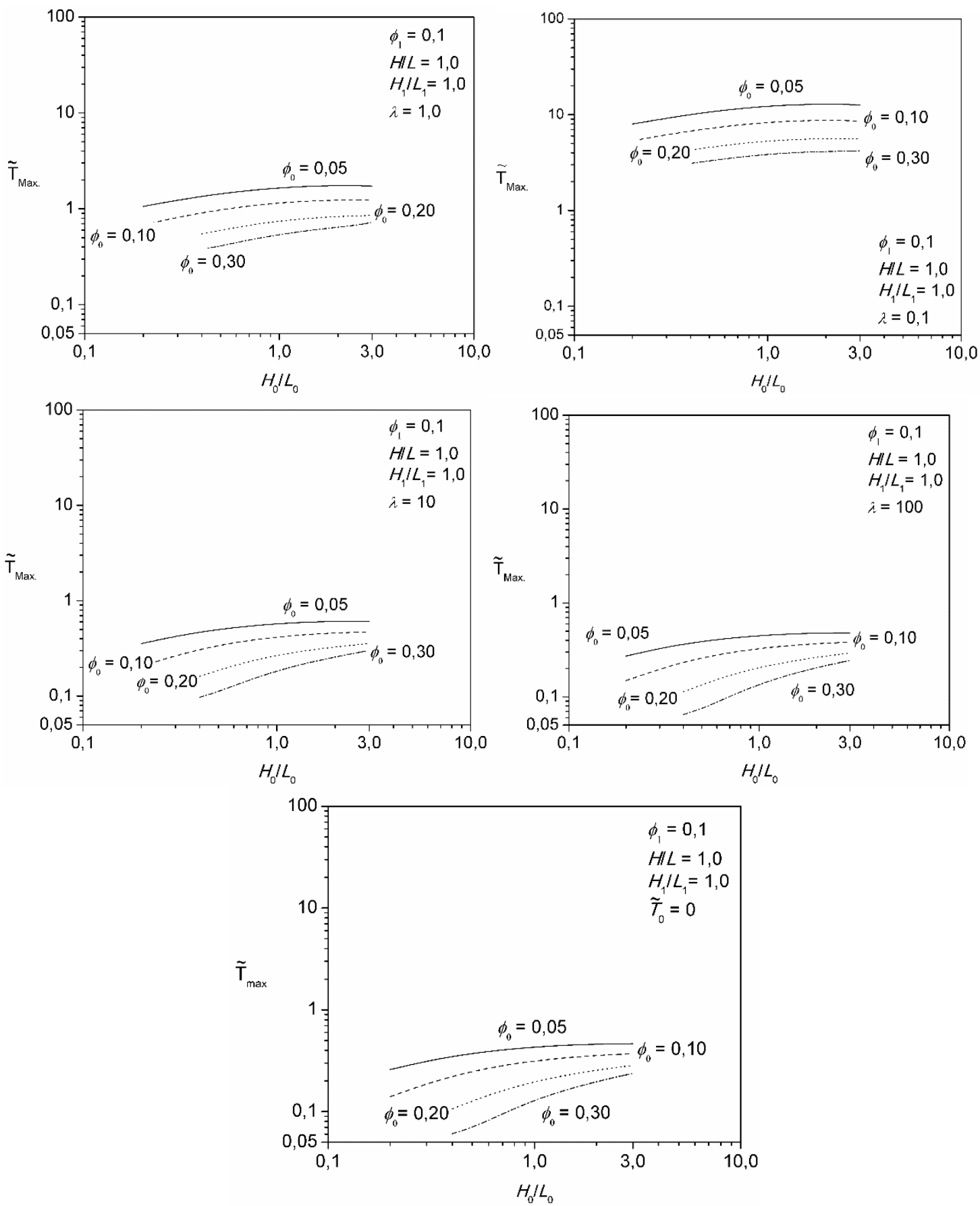

Figura 2: Comportamento da temperatura máxima do sistema $\left(\widetilde{T}_{\text {max }}\right)$ em função da razão de aspecto $H_{0} / L_{0}$ para diferentes condições de contorno nas paredes da cavidade e aleta, $\widetilde{T}_{0}=0 ; \Lambda=0,1 ; 1 ; 10 ; 100 e$ valores de $\phi_{0}$, considerando: $\phi_{1}=0,10 ; H / L=1 ; H_{1} / L_{l}=1$ 


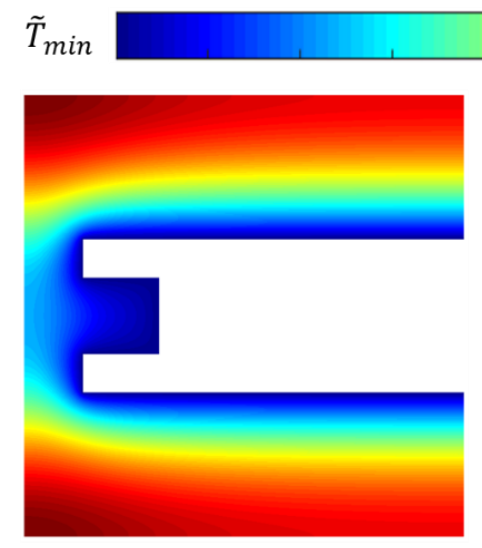

a)

$\left(\widetilde{T}_{\max }\right)_{\min }=0,0606$

$\left(H_{0} / L_{0}\right)_{0}=0,40$
$\tilde{T}_{\max }$

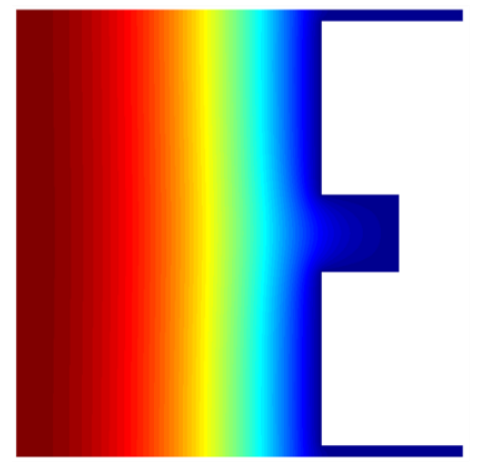

b)

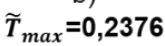

$H_{0} / L_{0}=3,00$

Figura 3: Campos de temperatura para: a) geometria ótima do sistema $\left.\left(H_{0} / L_{0}\right)_{o}=0,4 ; b\right)$ geometria de menor desempenho do sistema $H_{0} / L_{0}=3,0$, para $\tilde{T}_{0}=0 ; \phi_{0}=0,30 ; \phi_{1}=0,10 ; H / L=1 ; H_{1} / L_{l}=1$.
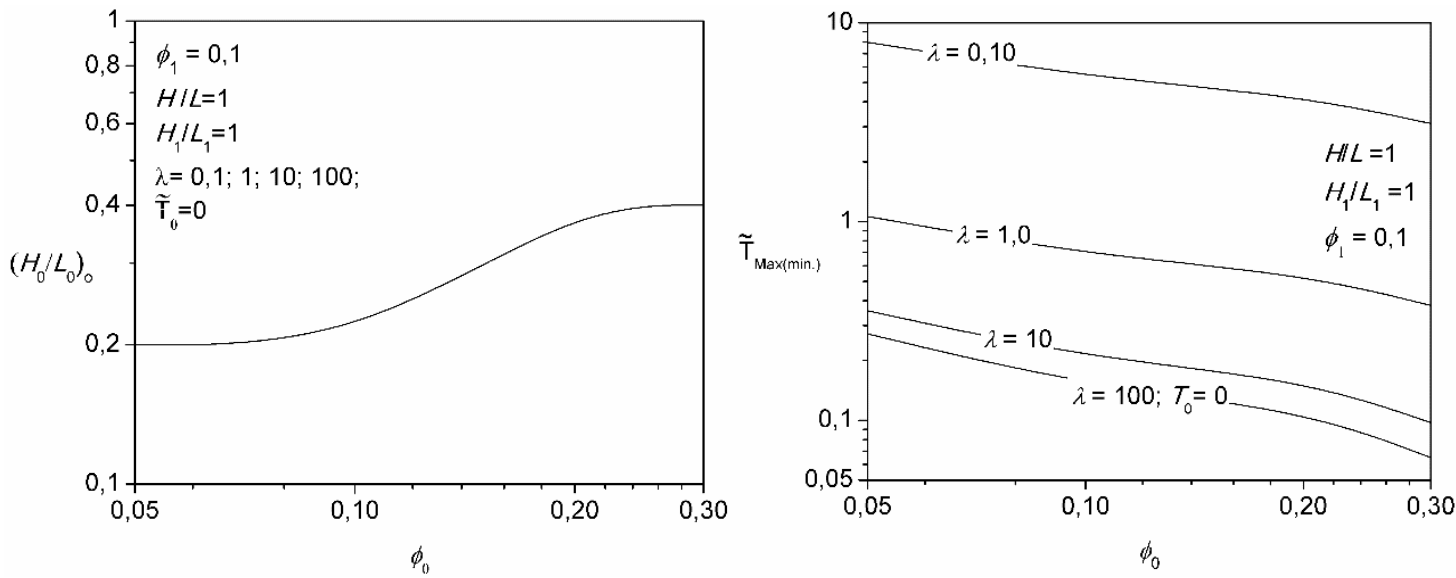

Figura 4: a) Comportamento de $\left(H_{0} / L_{0}\right)_{o}$ em função de $\left.\phi_{0} ; b\right)$ Comportamento de $\left(\widetilde{T}_{\text {max }}\right)_{\text {min }}$ em função de $\phi_{0}$, para : $\phi_{1}=0,10 ; H / L=1 ; H_{1} / L_{1}=1$.

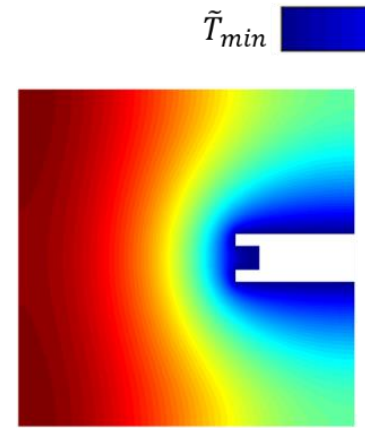

a)

$$
\begin{aligned}
\tilde{T}_{\max } & =0,3478 \\
\boldsymbol{\phi}_{\mathbf{0}} & =\mathbf{0 , 0 5}
\end{aligned}
$$

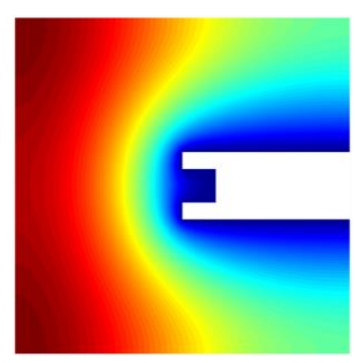

b)

$\tilde{T}_{\max }=0,2219$

$\phi_{0}=0,10$

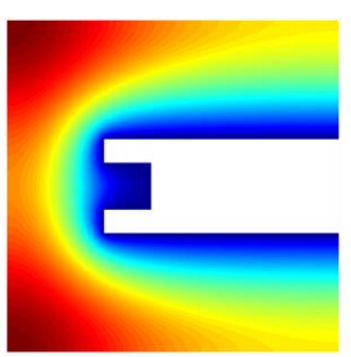

c)

$\tilde{T}_{\max }=0,1067$ $\phi_{0}=\mathbf{0 , 2 0}$
$\tilde{T}_{\max }$

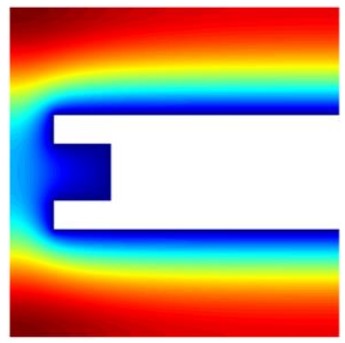

d)

$\tilde{T}_{\max }=0,0606$ $\phi_{0}=\mathbf{0 , 3 0}$

Figura 5: Campos de temperatura para: a) $\phi_{0}=0,05 ;$ b) $\phi_{0}=0,10 ;$ c) $\phi_{0}=0,20$ d) $\phi_{0}=0,30$; considerandose: $\widetilde{T}_{0}=0 ; \phi_{1}=0,10 ; H / L=1 ; H_{l} / L_{l}=1 ; H_{0} / L_{0}=0,40$. 


\section{CONCLUSÃO}

Este trabalho abordou a influência geométrica de cavidades aletadas em forma de 'E' inseridas em corpos sólidos com geração de calor. O método Design Construtal foi aplicado visando encontrar a geometria do sistema que maximizou seu desempenho térmico, minimizando a sua temperatura máxima $\left(\tilde{T}_{\text {max }}\right)$, através da variação do grau de liberdade $H_{0} / L_{0}$ para diferentes valores de $\phi_{0}\left(\phi_{0}=0,05 ; 0,10 ; 0,20 ; 0,30\right)$ e condições de contorno na parede da cavidade ( $\widetilde{T}_{0}=0$ e $\Lambda=0,1$; $1,0 ; 10 ; 100)$.

Inicialmente, foi elaborado o domínio computacional e sua discretização através de elementos triangulares não uniformes. A malha foi avaliada através do teste de qualidade de malha, de modo que a malha considerada satisfatória apresentou um desvio relativo adequado em relação a malha de maior número de volumes, justificando seu emprego no trabalho. Além disso, o modelo numérico adotado para o trabalho apresentou-se adequado do ponto de vista qualitativo (campos de temperatura).

O método Design Construtal mostrou-se adequado para a obtenção das configurações ótimas do sistema, uma vez que a temperatura máxima presente no corpo aquecido foi fortemente afetada pela variação do grau de liberdade $H_{0} / L_{0}$, tornando possível a obtenção de uma configuração que equilibrou a distribuição de temperaturas no sistema a favor da função objetivo. Além disso, constatou-se que os resultados ótimos obtidos apresentaram ganhos de performance consideráveis, apresentando um melhor rendimento térmico em relação aos casos de menor desempenho. Note-se ainda que os resultados estão de acordo com o princípio da ótima distribuição das imperfeições.

Como proposta de continuidade para este estudo, considera-se de suma importância avaliar os efeitos dos demais graus de liberdade do sistema $\left(H / L ; H_{0} / L_{0} ; H_{1} / L_{1}\right)$ de forma conjunta, uma vez que, segundo o Design Construtal, ao dar liberdade ao sistema pode-se chegar a resultados ainda mais satisfatórios.

\section{AGRADECIMENTOS}

Os autores F. S. F. Zinani, L. A. Isoldi, E. D. dos Santos e L. A. O. Rocha agradecem ao CNPq pela bolsa de produtividade em pesquisa (Processos: 307827/2018-6, 306012/2017-0, 306024/2017-9, 307847/2015-2). Os autores A. L. Razera, H. H. Horn e T. A. Hirt agradecem à CAPES e o autor G. Fraga agradece ao CNPq pelas bolsas de estudos. Os autores também agradecem a UFRGS, FURG e Unisinos que auxiliaram na elaboração deste estudo.

\section{REFERÊNCIAS BIBLIOGRÁFICAS}

1. Biserni C, Rocha LAO, Bejan A. Inverted fins: geometric optimization of the intrusion into a conducting wall. Int .J. Heat Mass Transf. 2004 Jun;47(12-13):2577-2586, doi: 10.1016/j.ijheatmasstransfer.2003.12.018

2. Biserni C, Rocha LAO, Stanescu G, Lorenzini E. Constructal H-shaped cavities according to Bejan's theory. Int J Heat Mass Transf. 2007 Jun;50(11-12):2132-2138, doi: 10.1016/j.ijheatmasstransfer.2006.11.006

3. Lorenzini GG, Biserni CC, Isoldi LA, dos Santos ED, Rocha LO. Constructal Design Applied to the Geometric Optimization of Y-shaped Cavities Embedded in a Conducting Medium. ASME. J. Electron. Packag. 2011 Dec;133(4), doi: 10.1115/1.4005296

4. Bejan A. Shape and structure, from engineering to nature. Cambridge University Press; 2000.

5. Bejan A. Constructal-theory network of conducting paths for cooling a heat generating volume. Int $\mathbf{J}$ Heat Mass Transf. 1997 Mar;40(4):799-816, doi: 10.1016/0017-9310(96)00175-5

6. Bejan A, Lorente S. Constructal theory of generation of config/uration in nature and engineering. J Appl Phys. 2006 Jun;100(4):5, doi: 10.1063/1.2221896

7. Bejan A, Lorente S. Design with Constructal theory. New Jersey: John Wiley \& Sons, Inc.; 2008.

8. Rocha LA., Lorenzini E, Biserni C. Geometric optimization of shapes on the basis of Bejan's Constructal theory. Int J Heat Mass Transf. 2005 Nov;32(10):1281-1288, doi: 10.1016/j.icheatmasstransfer.2005.07.010 
9. Lorenzini G, Rocha LAO, Biserni C., dos Santos ED, Isoldi LA. Constructal Design of Cavities Inserted Into a Cylindrical Solid Body. ASME. J. Heat Transf. 2012 May;134(7):071301-071301-6, doi: 10.1115/1.4006103.

10. Lorenzini G, Rocha LAO. Geometric optimization of TY-shaped cavity according to Constructal design. Int J Heat Mass Transf. 2009 Oct;52(21-22):4683-4688, doi: 10.1016/j.ijheatmasstransfer.2009.06.020

11. Xie Z, Chen, L, Sun F. Constructal optimization of twice Y-shaped assemblies of fins by taking maximum thermal resistance minimization as objective. Sci China Techn Sci. 2010 Sep;53(10):2756-2764, doi: 10.1007/s11431-010-4037-x

12. Lorenzini G, Biserni C, Link FB, Isoldi LA, Dos Santos ED, Rocha LAO. Constructal design of T-shaped cavity for several convective fluxes imposed at the cavity surfaces. J Eng Thermophys. 2013 Nov;22(4), 309-321, doi: 10.1134/S1810232813040048

13. Hajmohammadi MR, Abianeh VA, Moezzinajafabadi M, Daneshi M. Fork-shaped highly conductive pathways for maximum cooling in a heat generating piece. Appl Thermal Eng. 2013 Aug;61(2), 228-235, doi: 10.1016/j.applthermaleng.2013.08.001

14. Estrada EDSD, Gonzales GV, Dos Santos ED, Isoldi LA, Rocha, LAO. Constructal design aplicado à cavidades convectivas inseridas em um sólido cilíndrico. Scientia Plena, 2015 Dec;11(8):1-9, doi: 10.14808/sci.plena.2015.081325

15. Lorenzini G, Biserni C, Link FB, Dos Santos ED, Isoldi LA, Rocha LAO. Constructal design of isothermal X-shaped cavities. Thermal Sci. 2014 Dec;18(2):349-356, doi: 10.2298/TSCI120804005L

16. Biserni C, Dalpiaz FL, Fagundes TM, Rocha, LAO. Constructal design of T-shaped morphing fins coupled with a trapezoidal basement: a numerical investigation by means of exhaustive search and genetic algorithm. Int J Heat Mass Transf. 2017 Jun;109(1):73-81, doi: 10.1016/j.ijheatmasstransfer.2017.01.033 